\title{
Formal Predicate Calculus
}

Michael Meyling

May 24, 2013 
The source for this document can be found here:

http://www.qedeq.org/0_04_07/doc/math/qedeq_formal_logic_v1.xml

Copyright by the authors. All rights reserved.

If you have any questions, suggestions or want to add something to the list of modules that use this one, please send an email to the address mime@qedeq.org

The authors of this document are: Michael Meyling michael@meyling.com 


\section{Contents}

$\begin{array}{ll}\text { Summary } & 5\end{array}$

1 Language 7

1.1 Terms and Formulas . . . . . . . . . . . . 7

2 Axioms and Rules of Inference 11

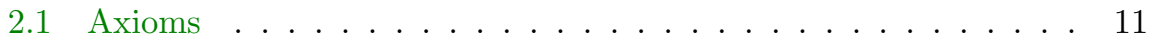

2.2 Rules of Inference . . . . . . . . . . . . . . . . . . . . 12

3 Propositional Calculus $\quad 15$

3.1 First Propositions . . . . . . . . . . . . . . 15

3.2 Deduction Theorem . . . . . . . . . . . . . . . . . . 16

3.3 Propositions about implication . . . . . . . . . . . . 17

3.4 Propositions about conjunction . . . . . . . . . . . . . 19

3.5 Propositions about disjunction . . . . . . . . . . . . 24

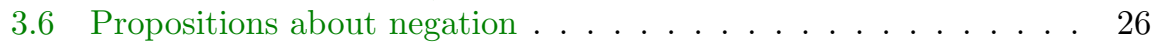

3.7 Mixing conjunction and disjunction. . . . . . . . . 29

Bibliography $\quad 33$

$\begin{array}{ll}\text { Index } & 33\end{array}$ 


\section{Summary}

In this text we present the development of predicate calculus in axiomatic form. The language of our calculus bases on the formalizations of D. Hilbert, W. Ackermann[3], P. S. Novikov[1], V. Detlovs and K. Podnieks[2]. New rules can be derived from the herein presented logical axioms and basic inference rules. Only these meta rules lead to a smooth flowing logical argumentation. For background informations see under https://dspace.lu.lv/dspace/handle/ 7/1308 [2] and http://en.wikipedia.org/wiki/Propositional_calculus. 


\section{Chapter 1}

\section{Language}

In this chapter we define a formal language to express mathematical propositions in a very precise way. Although this document describes a very formal approach to express mathematical content it is not sufficent to serve as a definition for an computer readable document format. Therefore such an extensive specification has to be done elsewhere. Here our choosen format is the Extensible Markup Language abbreviated XML. XML is a set of rules for encoding documents electronically. ${ }^{1}$ The according formal syntax specification can be found at http://www.qedeq.org/current/xml/qedeq.xsd. It specifies a complete mathematical document format that enables the generation of $\mathrm{LTT}_{\mathrm{E} X b o o k s}$ and makes automatic proof checking possible. Further syntax restrictions and some explanations can be found at http://www.qedeq.org/current/doc/project/ qedeq_logic_language_en.pdf.

Even this document is (or was generated) from an XML file that can be found here: http://wwww.qedeq.org/0_04_07/doc/math/qedeq_logic_v1.xml. But now we just follow the traditional mathematical way to present the elements of mathematical logic.

\subsection{Terms and Formulas}

We use the logical symbols $L=\{$ ' $\neg$ ', ' $\vee$ ', ' $\wedge$ ', '↔', ' $\rightarrow$ ', ' $\forall$ ', ' $\exists$ ' $\}$, the predicate constants $C=\left\{c_{i}^{k} \mid i, k \in \omega\right\}$, the function variables ${ }^{2} F=\left\{f_{i}^{k} \mid i, k \in\right.$ $\omega \wedge k>0\}$, the function constants ${ }^{3} H=\left\{h_{i}^{k} \mid i, k \in \omega\right\}$, the subject variables $V=\left\{v_{i} \mid i \in \omega\right\}$, as well as predicate variables $P=\left\{p_{i}^{k} \mid i, k \in \omega\right\}$. ${ }^{4}$ For the arity or rank of an operator we take the upper index. The set of predicate variables with zero arity is also called set of proposition variables or sentence letters: $A:=\left\{p_{i}^{0} \mid i \in \omega\right\}$. For subject variables we write short hand certain lower letters: $v_{1}=' u$ ', $v_{2}=' v ', v_{3}={ }^{\prime} w ', v_{4}=' x ', v_{5}={ }^{\prime} y ', v_{5}=' z$ '. Furthermore we use the following short notations: for the predicate variables $p_{1}^{n}=$ ' $\phi$ ' und $p_{2}^{n}=$ ' $\psi$ ', where the appropriate arity $n$ is calculated by counting the subsequent parameters, for the proposition variables $a_{1}=$ ' $A$ ', $a_{2}=$ ' $B$ ' and $a_{3}={ }^{\prime} C$ ',

\footnotetext{
${ }^{1}$ See http://www.w3.org/XML/ for more information.

${ }^{2}$ Function variables are used for a shorter notation. For example writing an identity proposition $x=y \rightarrow f(x)=f(y)$. Also this introduction prepares for the syntax extension for functional classes.

${ }^{3}$ Function constants are also introduced for convenience and are used for direct defined class functions. For example to define building of the power class operator, the union and intersection operator and the successor function. All these function constants can be interpreted as abbreviations.

${ }^{4} \mathrm{By} \omega$ we understand the natural numbers including zero. All involved symbols are pairwise disjoint. Therefore we can conclude for example: $f_{i}^{k}=f_{i^{\prime}}^{k^{\prime}} \rightarrow\left(k=k^{\prime} \wedge i=i^{\prime}\right)$ and $h_{i}^{k} \neq v_{j}$.
} 
for the function variables: $f_{1}^{n}=$ ' $f$ ' und $f_{2}^{n}={ }^{\prime} g$ ', where again the appropriate arity $n$ is calculated by counting the subsequent parameters. All binary propositional operators are written in infix notation. Parentheses surrounding groups of operands and operators are necessary to indicate the intended order in which operations are to be performed. E. g. for the operator $\wedge$ with the parameters $A$ and $B$ we write $(A \wedge B)$.

In the absence of parentheses the usual precedence rules determine the order of operations. Especially outermost parentheses are omitted. Also empty parentheses are stripped.

The operators have the order of precedence described below (starting with the highest).

$$
\begin{gathered}
\neg, \forall, \exists \\
\wedge \\
\vee \\
\rightarrow, \leftrightarrow
\end{gathered}
$$

The term term is defined recursively as follows:

1. Every subject variable is a term.

2. Let $i, k \in \omega$ and let $t_{1}, \ldots, t_{k}$ be terms. Then $h_{i}^{k}\left(t_{1}, \ldots, t_{k}\right)$ is a term and if $k>0$, so $f_{i}^{k}\left(t_{1}, \ldots, t_{k}\right)$ is a term too.

Therefore all zero arity function constants $\left\{h_{i}^{0} \mid i \in \omega\right\}$ are terms. They are called individual constants. ${ }^{5}$

We define a formula and the relations free and bound subject variable recursivly as follows:

1. Every proposition variable is a formula. Such formulas contain no free or bound subject variables.

2. If $p^{k}$ is a predicate variable with arity $k$ and $c^{k}$ is a predicate constant with arity $k$ and $t_{1}, t_{2}, \ldots, t_{k}$ are terms, then $p^{k}\left(t_{1}, t_{2}, \ldots t_{k}\right)$ and $c^{k}\left(t_{1}, t_{2}, \ldots, t_{k}\right)$ are formulas. All subject variables that occur at least in one of $t_{1}, t_{2}, \ldots, t_{k}$ are free subject variables. Bound subject variables does not occur. ${ }^{6}$

3. Let $\alpha, \beta$ be formulas in which no subject variables occur bound in one formula and free in the other. Then $\neg \alpha,(\alpha \wedge \beta),(\alpha \vee \beta),(\alpha \rightarrow \beta)$ and $(\alpha \leftrightarrow \beta)$ are also formulas. Subject variables which occur free (respectively bound) in $\alpha$ or $\beta$ stay free (respectively bound).

4. If in the formula $\alpha$ the subject variable $x_{1}$ occurs not bound ${ }^{7}$, then also $\forall x_{1} \alpha$ and $\exists x_{1} \alpha$ are formulas. The symbol $\forall$ is called universal quantifier and $\exists$ as existential quantifier.

Except for $x_{1}$ all free subject variables of $\alpha$ stay free. All bound subject variables are still bound and additionally $x_{1}$ is bound too.

All formulas that are only built by usage of 1 . and 3 . are called formulas of the propositional calculus.

\footnotetext{
${ }^{5}$ In an analogous manner subject variables might be defined as function variables of zero arity. Because subject variables play an important role they have their own notation.

${ }^{6}$ This second item includes the first one, which is only listed for clarity.

${ }^{7}$ This means that $x_{1}$ is free in the formula or does not occur at all.
} 
For each formula $\alpha$ the following proposition holds: the set of free subject variables is disjoint with the set of bound subject variables.. ${ }^{8}$

If a formula has the form $\forall x_{1} \alpha$ respectively $\exists x_{1} \alpha$ then the formula $\alpha$ is called the scope of the quantifier $\forall$ respectively $\exists$.

All formulas that are used to build up a formula by 1 . to 4 . are called part formulas.

${ }^{8}$ Other formalizations allow for example $\forall x_{1} \alpha$ also if $x_{1}$ occurs already bound within $\alpha$. Also propositions like $\alpha\left(x_{1}\right) \wedge\left(\forall x_{1} \beta\right)$ are allowed. In this formalizations free and bound are defined for a single occurrence of a variable. 


\section{Chapter 2}

\section{Axioms and Rules of Inference}

We now state the system of axioms for the propositional calculus and present the rules for obtaining new formulas from them.

\subsection{Axioms}

We just list the axioms without further explanations.

Axiom 1 (Implication Introduction). [axion: THEN-1]

$$
A \rightarrow(B \rightarrow A)
$$

Axiom 2 (Distribute Hypothesis over Implication). [axion:THEN-2]

$$
(A \rightarrow(B \rightarrow C)) \rightarrow((A \rightarrow B) \rightarrow(A \rightarrow C))
$$

Axiom 3 (Eliminate Conjunction Right). [axion:AnD-1]

$$
(A \wedge B) \rightarrow A
$$

Axiom 4 (Eliminate Conjunction Left). [axion:AnD-2]

$$
(A \wedge B) \rightarrow B
$$

Axiom 5 (Conjunction Introduction). [axion:AND-3]

$$
B \rightarrow(A \rightarrow(A \wedge B))
$$

Axiom 6 (Disjunction Introduction Right). [axion:0R-1]

$$
A \rightarrow(A \vee B)
$$

Axiom 7 (Disjunction Introduction Left). [axion:0R-2]

$$
A \rightarrow(B \vee A)
$$

Axiom 8 (Disjunction Elimination). [axiom:0R-3]

$$
(A \rightarrow C) \rightarrow((B \rightarrow C) \rightarrow((A \vee B) \rightarrow C))
$$


Axiom 9 (Negation Introduction). [axiom:NoT-1]

$$
(A \rightarrow B) \rightarrow((A \rightarrow \neg B) \rightarrow \neg A)
$$

Axiom 10 (Negation Elimination). [axiom:NoT-2]

$$
\neg A \rightarrow(A \rightarrow B)
$$

Axiom 11 (Excluded Middle). [axion: Nor-3]

$$
A \vee \neg A
$$

Axiom 12 (Equivalence Elimination right). [axiom:IFF-1]

$$
(A \leftrightarrow B) \rightarrow(A \rightarrow B)
$$

Axiom 13 (Equivalence Elimination left). [axiom:IfF-2]

$$
(A \leftrightarrow B) \rightarrow(B \rightarrow A)
$$

Axiom 14 (Equivalence Introduction). [axion:IFF-3]

$$
(A \rightarrow B) \rightarrow((B \rightarrow A) \rightarrow(A \leftrightarrow B))
$$

If something is true for all $x$, it is true for any specific $y$.

Axiom 15 (Universal Instantiation).

$$
\forall x \phi(x) \rightarrow \phi(y)
$$

If a predicate holds for some particular $y$, then there is an $x$ for which the predicate holds.

Axiom 16 (Existential Generalization).

$$
\phi(y) \rightarrow \exists x \phi(x)
$$

\subsection{Rules of Inference}

The following rules of inference enable us to obtain new true formulas from the axioms that are assumed to be true. From these new formulas we derive further formulas. So we can successively extend the set of true formulas.

Rule 1 (Add true formula).

Name: Add - Version: 0.01.00

Addition of an axiom, definition or already proven formula. We have to reference to the location of a true formula.

Rule 2 (Modus Ponens). [rule:modusponens]

Name: MP - Version: 0.01.00

If both formulas $\alpha$ and $\alpha \rightarrow \beta$ are true, then we can conclude that $\beta$ is true as well. 
Rule 3 (Replace Free Subject Variable). [rule:replacerree]

Name: SubstFree - Version: 0.01.00

We start with a true formula. A free subject variable may be replaced by an arbitrary term, provided that the substituted term contains no subject variable that have a bound occurrence in the original formula. All occurrences of the free variable must be simultaneously replaced.

The prohibition to use subject variables within the term that occur bound in the original formula has two reasons. First it ensures that the resulting formula is well-formed. Secondly it preserves the validity of the formula. Let us look at the following derivation.

$$
\begin{array}{lll}
\forall x \exists y \phi(x, y) & \rightarrow \exists y \phi(z, y) & \text { with axiom } 15 \\
\forall x \exists y \phi(x, y) & \rightarrow \exists y \phi(y, y) & \begin{array}{l}
\text { forbidden replacement: } z \text { in } y, \text { despite } y \text { is } \\
\text { already bound }
\end{array} \\
\forall x \exists y x \neq y & \rightarrow \exists y y \neq y \quad \begin{array}{l}
\text { replace } \phi \text { by } \neq
\end{array}
\end{array}
$$

This last proposition is not valid in many models.

Rule 4 (Rename Bound Subject Variable). [rule:renameBound]

Name: Rename - Version: 0.01.00

We may replace a bound subject variable occurring in a formula by any other subject variable, provided that the new variable occurs not free in the original formula. If the variable to be replaced occurs in more than one scope, then the replacement needs to be made in one scope only.

Rule 5 (Replace Predicate Variable). [rule:replacePred]

Name: SubstPred - Version: 0.01.00

Let $\alpha$ be a true formula that contains a predicate variable $p$ of arity $n$, let $x_{1}$, $\ldots, x_{n}$ be pairwise different subject variables and let $\beta\left(x_{1}, \ldots, x_{n}\right)$ be a formula where $x_{1}, \ldots, x_{n}$ are not bound. The formula $\beta\left(x_{1}, \ldots, x_{n}\right)$ must not contain all $x_{1}, \ldots, x_{n}$ as free subject variables. Furthermore it can also have other subject variables either free or bound.

If the following conditions are fulfilled, then a replacement of all occurrences of $p\left(t_{1}, \ldots, t_{n}\right)$ each with appropriate terms $t_{1}, \ldots, t_{n}$ in $\alpha$ by $\beta\left(t_{1}, \ldots, t_{n}\right)$ results in another true formula.

- the free variables of $\beta\left(x_{1}, \ldots, x_{n}\right)$ without $x_{1}, \ldots, x_{n}$ do not occur as bound variables in $\alpha$

- each occurrence of $p\left(t_{1}, \ldots, t_{n}\right)$ in $\alpha$ contains no bound variable of $\beta\left(x_{1}, \ldots, x_{n}\right)$

- the result of the substitution is a well-formed formula

See III $\S 5$ in [3].

We can think in the same lines as by rule 3 . The prohibition to use additional subject variables within the replacement formula that occur bound in the original formula assurs that the resulting formula is well-formed. Furthermore it preserves the validity of the formla. Take a look at the following derivation.

$$
\begin{array}{rll}
\phi(x) & \rightarrow \exists y \phi(y) & \text { with axiom } 16 \\
(\exists y y=y) \wedge \phi(x) & \rightarrow \exists y \phi(y) & \\
\exists y(y=y \wedge \phi(x)) & \rightarrow \exists y \phi(y) & \\
\exists y(y=y \wedge x \neq y) & \rightarrow \exists y y \neq y \quad \text { forbidden replacment: } \phi(x) \text { by } x \neq y \\
\exists y x \neq y & \rightarrow \exists y y \neq y & \text { despite } y \text { is already bound }
\end{array}
$$


The last proposition is not valid in many models.

Analogous to rule 5 we can replace function variables too.

Rule 6 (Replace Function Variable). [rule:replacefunct]

Name: SubstFun - Version: 0.01.00

Let $\alpha$ be an already proved formula that contains a function variable $\sigma$ of arity $n$, let $x_{1}, \ldots, x_{n}$ be pairwise different subject variables and let $\tau\left(x_{1}, \ldots, x_{n}\right)$ be an arbitrary term where $x_{1}, \ldots, x_{n}$ are not bound. The term $\tau\left(x_{1}, \ldots, x_{n}\right)$ must not contain all $x_{1}, \ldots, x_{n}$ as free subject variables. Furthermore it can also have other subject variables either free or bound.

If the following conditions are fulfilled we can obtain a new true formula by replacing each occurrence of $\sigma\left(t_{1}, \ldots, t_{n}\right)$ with appropriate terms $t_{1}, \ldots, t_{n}$ in $\alpha$ by $\tau\left(t_{1}, \ldots, t_{n}\right)$.

- the free variables of $\tau\left(x_{1}, \ldots, x_{n}\right)$ without $x_{1}, \ldots, x_{n}$ do not occur as bound variables in $\alpha$

- each occurrence of $\sigma\left(t_{1}, \ldots, t_{n}\right)$ in $\alpha$ contains no bound variable of $\tau\left(x_{1}, \ldots, x_{n}\right)$

- the result of the substitution is a well-formed formula

Rule 7 (Universal Generalization). [rule: universalGeneral ization]

Name: Universal - Version: 0.01.00

If $\alpha \rightarrow \beta\left(x_{1}\right)$ is a true formula and $\alpha$ does not contain the subject variable $x_{1}$, then $\alpha \rightarrow\left(\forall x_{1}\left(\beta\left(x_{1}\right)\right)\right)$ is a true formula too.

Rule 8 (Existential Generalization).

Name: Existential - Version: 0.01.00

If $\alpha\left(x_{1}\right) \rightarrow \beta$ is already proved to be true and $\beta$ does not contain the subject variable $x_{1}$, then $\left(\exists x_{1} \alpha\left(x_{1}\right)\right) \rightarrow \beta$ is also a true formula. 


\section{Chapter 3}

\section{Propositional Calculus}

In this chapter we introduce an importent new inference rule and develop the traditional results of propositional calculus.

\subsection{First Propositions}

Here we draw the first conclusions.

Proposition 1.

[proposition: implicationReflexive1]

$$
A \rightarrow A
$$

Proof.

(1) $A \rightarrow(B \rightarrow A)$

(2) $(A \rightarrow(B \rightarrow C)) \rightarrow((A \rightarrow B) \rightarrow(A \rightarrow C)) \quad$ Add axiom 1

(3) $A \rightarrow(B \vee A) \quad$ Add axiom 7

(4) $A \rightarrow((B \vee A) \rightarrow A) \quad$ SubstPred $B$ by $B \vee A$ in (1)

$(5)(A \rightarrow((B \vee A) \rightarrow C)) \rightarrow((A \rightarrow(B \vee A)) \rightarrow$ SubstPred $B$ by $B \vee A$ in (2) $(A \rightarrow C))$

(6) $(A \rightarrow((B \vee A) \rightarrow A)) \rightarrow((A \rightarrow(B \vee A)) \rightarrow \operatorname{SubstPred} C$ by $A$ in (5) $(A \rightarrow A))$

(7) $(A \rightarrow(B \vee A)) \rightarrow(A \rightarrow A)$

(8) $A \rightarrow A$

MP (6), (4)

MP (7), (3)

Proposition 2. [proposition: implication2]

$$
(A \vee A) \rightarrow A
$$

Proof.

(1) $A \rightarrow A$

(2) $(A \rightarrow C) \rightarrow((B \rightarrow C) \rightarrow((A \vee B) \rightarrow C))$

Add proposition 1

(3) $(A \rightarrow C) \rightarrow((A \rightarrow C) \rightarrow((A \vee A) \rightarrow C))$

Add axiom 8

(4) $(A \rightarrow A) \rightarrow((A \rightarrow A) \rightarrow((A \vee A) \rightarrow A))$

SubstPred $B$ by $A$ in (2)

(5) $(A \rightarrow A) \rightarrow((A \vee A) \rightarrow A)$

(6) $(A \vee A) \rightarrow A$ 
Proposition 3. [proposition: implicationo3]

$$
(A \vee B) \rightarrow(B \vee A)
$$

Proof.

(1) $A \rightarrow(A \vee B)$

Add axiom 6

(2) $A \rightarrow(B \vee A)$

(3) $(A \rightarrow C) \rightarrow((B \rightarrow C) \rightarrow((A \vee B) \rightarrow C))$

Add axiom 7

(4) $D \rightarrow(D \vee B)$

Add axiom 8

(5) $(A \rightarrow(C \vee A))$ $(C \vee A)))$

(6) $D \rightarrow(D \vee A)$

SubstPred $A$ by $D$ in (1)

(7) $(A \rightarrow(B \vee A))$ $(B \vee A)))$

(8) $(B \rightarrow(B \vee A)) \rightarrow((A \vee B) \rightarrow(B \vee A))$

(9) $B \rightarrow(B \vee A)$

$(10)(A \vee B) \rightarrow(B \vee A)$

Proposition 4.

[proposition: implication 04$]$

$$
\neg(A \wedge \neg A)
$$

Proof.

(1) $(A \wedge B) \rightarrow A$

(2) $(A \wedge B) \rightarrow B$

(3) $(A \rightarrow B) \rightarrow((A \rightarrow \neg B) \rightarrow \neg A)$

Add axiom 3

(4) $(A \wedge \neg A) \rightarrow A$

dd axiom

(5) $(A \wedge \neg A) \rightarrow \neg A$

SubstPred $B$ by $\neg A$ in (1)

(6) $((A \wedge \neg A) \rightarrow B) \rightarrow(((A \wedge \neg A) \rightarrow \neg B) \rightarrow$ SubstPred $A$ by $A \wedge \neg A$ in (3) $\neg(A \wedge \neg A))$

(7) $((A \wedge \neg A) \rightarrow A) \rightarrow(((A \wedge \neg A) \rightarrow \neg A) \rightarrow \neg(A \wedge \neg A))$ SubstPred $B$ by $A$ in (6)

(8) $((A \wedge \neg A) \rightarrow \neg A) \rightarrow \neg(A \wedge \neg A)$

(9) $\neg(A \wedge \neg A)$

MP (7), (4)

MP (8), (5)

\subsection{Deduction Theorem}

We prove the deduction theorem. This leads to the new rule Conditional Proof.

If we can prove $B$ by assuming $A$ as a hypothesis then we have proved $A \rightarrow B$. This reasoning is justified by the so-called deduction theorem. The deduction theorem holds for all first-order theories with the usual deductive systems for first-order logic. However our use of proposition variables and substitution rules make difficulties. We have to restrict the allowed inference rules to get a simular result.

Rule 9. [rule:CP]

Name: CP - Version: 0.02.00

We have the well-formed formula $\alpha$ and add it as a new proof line. Now we modify the existing inference rules. We can add a further proof line $\beta$ if $\alpha \rightarrow \beta$ 
is a well-formed formula and the usage of a previous inference rule with the following restrictions justifies the addition: for rule 3 occurs the replaced free variable not in $\alpha$, for rule 5 occurs the replaced predicate variable not in $\alpha$, for rule 6 occurs the replaced function variable not in $\alpha$.

Based on: axiom 1 axiom 2 The following rules have to be extended.

Name: MP - Version: 0.02.00 - Old Version: 0.01.00

See rule 9 .

Name: Add - Version: 0.02.00 - Old Version: 0.01.00

See rule 9 .

Name: Rename - Version: 0.02.00 - Old Version: 0.01.00

See rule 9 .

Name: SubstFree - Version: 0.02.00 - Old Version: 0.01.00

See rule 9.

Name: SubstPred - Version: 0.02.00 - Old Version: 0.01.00

See rule 9.

Name: SubstFun - Version: 0.02.00 - Old Version: 0.01.00

See rule 9.

Name: Universal - Version: 0.02.00 - Old Version: 0.01.00

See rule 9 .

Name: Existential - Version: 0.02.00 - Old Version: 0.01.00

See rule 9 .

Proof. TODO 20110613 m31

The deduction theorem enables us to prove propositions more easier in the next sections.

\subsection{Propositions about implication}

We use rule 9 to derive more propositions containing only the implication operator.

Proposition 5.

$$
(A \rightarrow(A \rightarrow B)) \rightarrow(A \rightarrow B)
$$

Proof.

(1) $\quad A \rightarrow(A \rightarrow B)$

Hypothesis

(2)

Conditional Proof

(3) $\quad A \rightarrow B$

$A$
$A \rightarrow B$

$B$

Hypothesis

$(4)$

(5) $A \rightarrow B$

MP (1), (2)

(6) $(A \rightarrow(A \rightarrow B)) \rightarrow(A \rightarrow B)$

Conclusion

Conclusion 
Proposition 6. [proposition: imp1ication11]

$$
((A \rightarrow B) \rightarrow(A \rightarrow C)) \rightarrow(A \rightarrow(B \rightarrow C))
$$

Proof.

(1) $A \rightarrow(B \rightarrow A)$

(2) $D \rightarrow(B \rightarrow D)$

(3) $D \rightarrow(A \rightarrow D)$

(4) $B \rightarrow(A \rightarrow B)$

Conditional Proof

(5) $\quad(A \rightarrow B) \rightarrow(A \rightarrow C)$ Conditional Proof

(6)

(12) $\quad A \rightarrow(B \rightarrow C)$

$(13)((A \rightarrow B) \rightarrow(A \rightarrow C)) \rightarrow(A \rightarrow(B \rightarrow C))$

Proposition 7.

$$
(A \rightarrow B) \rightarrow((B \rightarrow C) \rightarrow(A \rightarrow C))
$$

Proof.

(1)

(2)

(6)

(7) $(B \rightarrow C) \rightarrow(A \rightarrow C)$

$(8)(A \rightarrow B) \rightarrow((B \rightarrow C) \rightarrow(A \rightarrow C))$

Proposition 8. [proposition: implication13]

$$
(A \rightarrow(B \rightarrow C)) \rightarrow(B \rightarrow(A \rightarrow C))
$$

Proof.

Conditional Proof

(1) $\quad A \rightarrow(B \rightarrow C)$

Conditional Proof

$(2)$

$B$

Conditional Proof
Add axiom 1

SubstPred $A$ by $D$ in (1)

SubstPred $B$ by $A$ in (2)

SubstPred $D$ by $B$ in (3)

Hypothesis

Hypothesis

Hypothesis

MP (4), (7)

MP (5), (8)

MP (9), (6)

Conclusion

Conclusion

Conclusion
Hypothesis

Hypothesis

Hypothesis

MP (1), (3)

MP (2), (4)

Conclusion

Conclusion

Conclusion
Hypothesis

Hypothesis 

(3)
(5)
$A$
Hypothesis
MP (1), (3)
MP (4), (2)
(6) $\quad A \rightarrow C$
$B \rightarrow C$
Conclusion
(7) $B \rightarrow(A \rightarrow C)$
(8) $(A \rightarrow(B \rightarrow C)) \rightarrow(B \rightarrow(A \rightarrow C))$
Conclusion
Conclusion

\subsection{Propositions about conjunction}

We use rule 9 to derive more propositions containing the conjunction operator.

Proposition 9. [proposition:implication14]

$$
A \rightarrow(A \wedge A)
$$

Proof.
(1) $B \rightarrow(A \rightarrow(A \wedge B))$
Add axiom 5
(2) $A \rightarrow(A \rightarrow(A \wedge A))$
SubstPred $B$ by $A$ in (1)
(3) $\quad A$
(4) $\quad A \rightarrow(A \wedge A)$
(5) $\quad A \wedge A$
(6) $A \rightarrow(A \wedge A)$

Proposition 10. [proposition: AnD-3b]

$$
A \rightarrow(B \rightarrow(A \wedge B))
$$

Proof.
(1) $(A \rightarrow(B \rightarrow C)) \rightarrow(B \rightarrow(A \rightarrow C))$
(2) $(A \rightarrow(D \rightarrow C)) \rightarrow(D \rightarrow(A \rightarrow C))$
Add proposition 8
$(3)(B \rightarrow(D \rightarrow C)) \rightarrow(D \rightarrow(B \rightarrow C))$
SubstPred $B$ by $D$ in (1)
(4) $(B \rightarrow(A \rightarrow C)) \rightarrow(A \rightarrow(B \rightarrow C))$
SubstPred $A$ by $B$ in (2)
(5) $(B \rightarrow(A \rightarrow(A \wedge B))) \rightarrow(A \rightarrow(B \rightarrow(A \wedge B)))$
SubstPred $D$ by $A$ in (3)
(6) $B \rightarrow(A \rightarrow(A \wedge B))$
(7) $A \rightarrow(B \rightarrow(A \wedge B))$
Add axiom 5
MP (5), (6)

Proposition 11. [proposition: imp1ication15]

$$
((A \rightarrow B) \wedge(B \rightarrow C)) \rightarrow(A \rightarrow C)
$$

Proof.
(1) $(A \wedge B) \rightarrow A$
(2) $(A \wedge(B \rightarrow C)) \rightarrow A$
(3) $((A \rightarrow B) \wedge(B \rightarrow C)) \rightarrow(A \rightarrow B)$
(4) $(A \wedge B) \rightarrow B$
(5) $(A \wedge(B \rightarrow C)) \rightarrow(B \rightarrow C)$

Add axiom 3

SubstPred $B$ by $B \rightarrow C$ in (1)

SubstPred $A$ by $A \rightarrow B$ in (2)

Add axiom 4

SubstPred $B$ by $B \rightarrow C$ in (4) 

(6) $((A \rightarrow B) \wedge(B \rightarrow C)) \rightarrow(B \rightarrow C) \quad$ SubstPred $A$ by $A \rightarrow B$ in (5) Conditional Proof
$(7) \quad(A \rightarrow B) \wedge(B \rightarrow C)$
(8) $\quad A \rightarrow B$
(9) $B \rightarrow C$
$(10) \quad(A \rightarrow B) \rightarrow((B \rightarrow C) \rightarrow(A \rightarrow C))$
$(11) \quad(B \rightarrow C) \rightarrow(A \rightarrow C)$
Hypothesis
MP (3), (7)
MP (6), (7)
(12) $A \rightarrow C$
Add proposition 7
MP (10), (8)
(13) $((A \rightarrow B) \wedge(B \rightarrow C)) \rightarrow(A \rightarrow C)$
MP (11), (9)
Conclusion

Proposition 12.

$$
(A \rightarrow B) \rightarrow((A \rightarrow C) \rightarrow(A \rightarrow(B \wedge C)))
$$

Proof.
(1) $B \rightarrow(A \rightarrow(A \wedge B))$
(2) $C \rightarrow(A \rightarrow(A \wedge C))$
Add axiom 5
(3) $C \rightarrow(B \rightarrow(B \wedge C))$
SubstPred $B$ by $C$ in (1) Conditional Proof
(4) $\quad A \rightarrow B$
Conditional Proof
(5) $A \rightarrow C$
Conditional Proof
(6) A
$\begin{array}{ll}(7) & C \\ (8) & B \rightarrow(B \wedge C)\end{array}$
(12) $(A \rightarrow C) \rightarrow(A \rightarrow(B \wedge C))$
(13) $(A \rightarrow B) \rightarrow((A \rightarrow C) \rightarrow(A \rightarrow(B \wedge C)))$
SubstPred $A$ by $B$ in (2)
Hypothesis
Hypothesis
Hypothesis
MP (5), (6)
MP (3), (7)
MP (4), (6)
MP (8), (9)
Conclusion
Conclusion
Conclusion

Proposition 13.

$$
(A \rightarrow B) \rightarrow((A \wedge C) \rightarrow(B \wedge C))
$$

Proof.
(1) $(A \wedge B) \rightarrow A$
(2) $(A \wedge C) \rightarrow A$
(3) $(A \wedge B) \rightarrow B$
(4) $(A \wedge C) \rightarrow C$
(5) $B \rightarrow(A \rightarrow(A \wedge B))$
(6) $C \rightarrow(A \rightarrow(A \wedge C))$
(7) $C \rightarrow(B \rightarrow(B \wedge C))$
Add axiom 3
SubstPred $B$ by $C$ in (1)
Add axiom 4
SubstPred $B$ by $C$ in (3)
Add axiom 5
SubstPred $B$ by $C$ in (5)
Conditional Proof
$A \rightarrow B$
Conditional Proof
(9) $A \wedge C$
(10) $A$
$A$
$B$
SubstPred $A$ by $B$ in (6)
(12)
C
Hypothesis
Hypothesis
MP (2), (9)
MP (8), (10)
MP (4), (9) 

(13)
$B \rightarrow(B \wedge C)$
(14) $B \wedge C$
MP (7), (12)
MP (13), (11)
(15) $(A \wedge C) \rightarrow(B \wedge C)$
$(16)(A \rightarrow B) \rightarrow((A \wedge C) \rightarrow(B \wedge C))$
Conclusion
Conclusion

Proposition 14. [proposition: implication19]

$$
(A \wedge B) \rightarrow(B \wedge A)
$$

Proof.
(1) $B \rightarrow(A \rightarrow(A \wedge B))$
(2) $C \rightarrow(A \rightarrow(A \wedge C))$
Add axiom 5
(3) $C \rightarrow(B \rightarrow(B \wedge C))$
(4) $A \rightarrow(B \rightarrow(B \wedge A))$
(5) $(A \wedge B) \rightarrow A$
(6) $(A \wedge B) \rightarrow B$
Conditional Proof
(7) $A \wedge B$
(8) $A$
(9) $B \rightarrow(B \wedge A)$
(10) $B$
(11) $B \wedge A$
(12) $(A \wedge B) \rightarrow(B \wedge A)$
SubstPred $B$ by $C$ in (1)
SubstPred $A$ by $B$ in (2)
SubstPred $C$ by $A$ in (3)
Add axiom 3
Add axiom 4
Hypothesis
MP (5), (7)
MP (4), (8)
MP (6), (7)
MP (9), (10)
Conclusion

Proposition 15. [proposition: implication20]

$$
(A \rightarrow(B \rightarrow C)) \rightarrow((A \wedge B) \rightarrow C)
$$

Proof.

$\begin{array}{ccc} & \text { Conditional Proof } \\ (1) & A \rightarrow(B \rightarrow C) & \\ & \text { Conditional Proof } & \\ (2) & A \wedge B & \text { Hypothesis } \\ (3) & (A \wedge B) \rightarrow A & \text { Add axiom 3 } \\ (4) & A \wedge \text { MP (3), (2) } \\ (5) & (A \wedge B) \rightarrow B & \text { Add axiom 4 } \\ (6) & B & \text { MP (5), (2) } \\ (7) & B \rightarrow C & \text { MP (1), (4) } \\ (8) & C & \text { MP (7), (6) } \\ (9) & (A \wedge B) \rightarrow C & \text { Conclusion } \\ (10) & (A \rightarrow(B \rightarrow C)) \rightarrow((A \wedge B) \rightarrow C) & \text { Conclusion }\end{array}$

Proposition 16.

$$
((A \wedge B) \rightarrow C) \rightarrow(A \rightarrow(B \rightarrow C))
$$

Proof. 


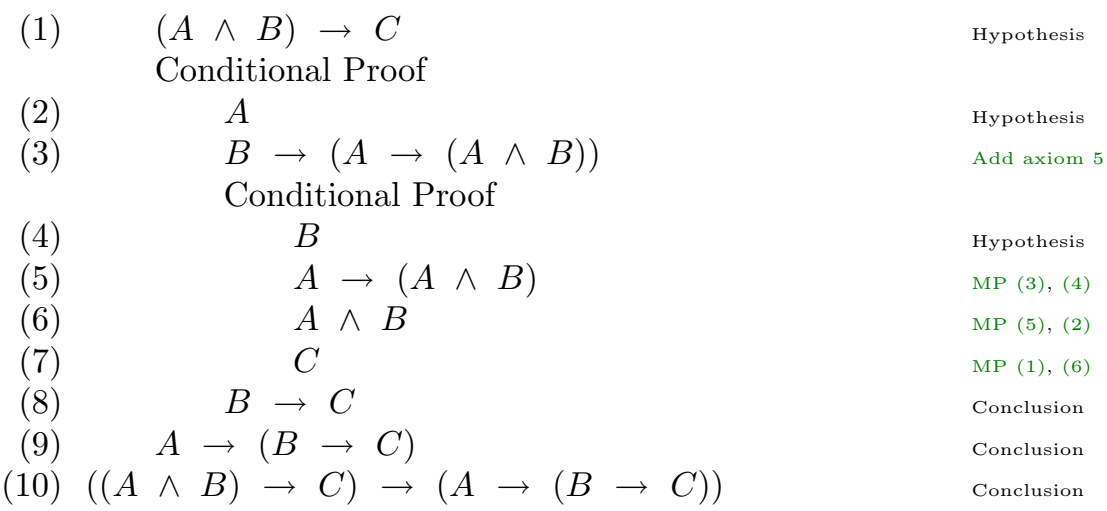

Proposition 17. [proposition:implication25]

$$
((A \rightarrow B) \wedge(A \rightarrow C)) \rightarrow(A \rightarrow(B \wedge C))
$$

Proof.
(1) $(A \wedge B) \rightarrow A$
(2) $(A \wedge C) \rightarrow A$
(3) $((A \rightarrow B) \wedge C) \rightarrow(A \rightarrow B)$
(4) $((A \rightarrow B) \wedge(A \rightarrow C)) \rightarrow(A \rightarrow B)$
(5) $(A \wedge B) \rightarrow B$
(6) $(A \wedge C) \rightarrow C$
(7) $((A \rightarrow B) \wedge C) \rightarrow C$
(8) $((A \rightarrow B) \wedge(A \rightarrow C)) \rightarrow(A \rightarrow C)$
(9) $B \rightarrow(A \rightarrow(A \wedge B))$
(10) $C \rightarrow(A \rightarrow(A \wedge C))$
(11) $C \rightarrow(B \rightarrow(B \wedge C))$
Conditional Proof
(12) $(A \rightarrow B) \wedge(A \rightarrow C)$
(13) $\quad A \rightarrow B$
(14) $A \rightarrow C$
Conditional Proof
$\begin{array}{ll}(15) & A \\ (16) & B\end{array}$
(17) $C$

$$
\begin{aligned}
& A \\
& B \\
& C \\
& B \rightarrow(B \wedge C)
\end{aligned}
$$
(19) $B \wedge C$
(20) $\quad A \rightarrow(B \wedge C)$
$(21)((A \rightarrow B) \wedge(A \rightarrow C)) \rightarrow(A \rightarrow(B \wedge C))$

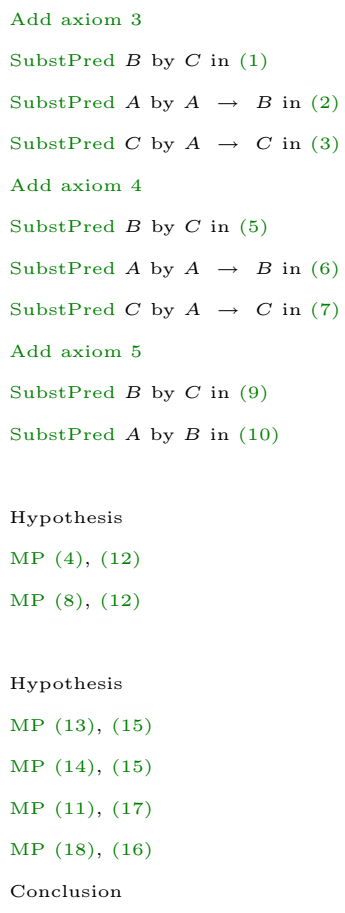

Proposition 18. [proposition:implication26]

$$
(A \rightarrow(B \wedge C)) \rightarrow((A \rightarrow B) \wedge(A \rightarrow C))
$$

Proof.

(1) $(A \wedge B) \rightarrow A$

(2) $(A \wedge C) \rightarrow A$

Add axiom 3

(3) $(B \wedge C) \rightarrow B$

(4) $(A \wedge B) \rightarrow B$ 

(5) $(A \wedge C) \rightarrow C$
(6) $(B \wedge C) \rightarrow C$
(7) $B \rightarrow(A \rightarrow(A \wedge B))$
SubstPred $B$ by $C$ in (4)
SubstPred $A$ by $B$ in (5)
(8) $C \rightarrow(A \rightarrow(A \wedge C))$
Add axiom 5
(9) $C \rightarrow((A \rightarrow B) \rightarrow((A \rightarrow B) \wedge C))$
SubstPred $B$ by $C$ in (7)

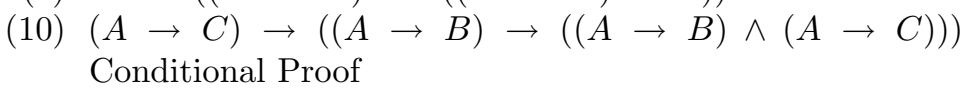
(11) $\quad A \rightarrow(B \wedge C)$
Conditional Proof
$\begin{array}{ll}(12) & A \\ (13) & B \wedge C \\ (14) & B\end{array}$
(14) $\quad A \stackrel{B}{\rightarrow} B$
SubstPred $A$ by $A \rightarrow B$ in (8)
Conditional Proof
$\begin{array}{ll}(16) & A \\ \text { (17) } & B \wedge C\end{array}$
(18) $\quad C$
(19) $\quad A \rightarrow C$
(20) $\quad(A \rightarrow B) \rightarrow((A \rightarrow B) \wedge(A \rightarrow C))$
SubstPred $C$ by $A \rightarrow C$ in (9)
(21) $\quad(A \rightarrow B) \wedge(A \rightarrow C)$
$(22)(A \rightarrow(B \wedge C)) \rightarrow((A \rightarrow B) \wedge(A \rightarrow C))$
Hypothesis
Hypothesis
MP (11), (12)
MP (3), (13)
Conclusion
Hypothesis
MP (11), (16)
MP (6), (17)
Conclusion
MP (10), (19)
MP (20), (15)
Conclusion

Proposition 19.

$$
((A \wedge B) \wedge C) \rightarrow(A \wedge(B \wedge C))
$$

Proof.

(1) $(A \wedge B) \rightarrow A$

(2) $(A \wedge C) \rightarrow A$

(3) $((A \wedge B) \wedge C) \rightarrow(A \wedge B)$

(4) $(A \wedge B) \rightarrow B$

(5) $(A \wedge C) \rightarrow C$

(6) $((A \wedge B) \wedge C) \rightarrow C$

(7) $B \rightarrow(A \rightarrow(A \wedge B))$

(8) $C \rightarrow(A \rightarrow(A \wedge C))$

(9) $C \rightarrow(B \rightarrow(B \wedge C))$

(10) $(B \wedge C) \rightarrow(A \rightarrow(A \wedge(B \wedge C)))$

Conditional Proof

(11) $\quad(A \wedge B) \wedge C$

(12) $A \wedge B$

(13) $\quad A$

(14) $B$

(15) $C$

(16) $B \rightarrow(B \wedge C)$

(17) $B \wedge C$

(18) $\quad A \rightarrow(A \wedge(B \wedge C))$

(19) $\quad A \wedge(B \wedge C)$

$(20)((A \wedge B) \wedge C) \rightarrow(A \wedge(B \wedge C))$

Add axiom 3

SubstPred $B$ by $C$ in (1)

SubstPred $A$ by $A \wedge B$ in (2)

Add axiom 4

SubstPred $B$ by $C$ in (4)

SubstPred $A$ by $A \wedge B$ in (5)

Add axiom 5

SubstPred $B$ by $C$ in (7)

SubstPred $A$ by $B$ in (8)

SubstPred $B$ by $B \wedge C$ in (7)

Hypothesis

MP (3), (11)

MP (1), (12)

MP (4), (12)

MP (6), (11)

MP (9), (15)

MP (16), (14)

MP (10), (17)

MP (18), (13)

Conclusion

Proposition 20.

$$
(A \wedge(B \wedge C)) \rightarrow((A \wedge B) \wedge C)
$$


Proof.
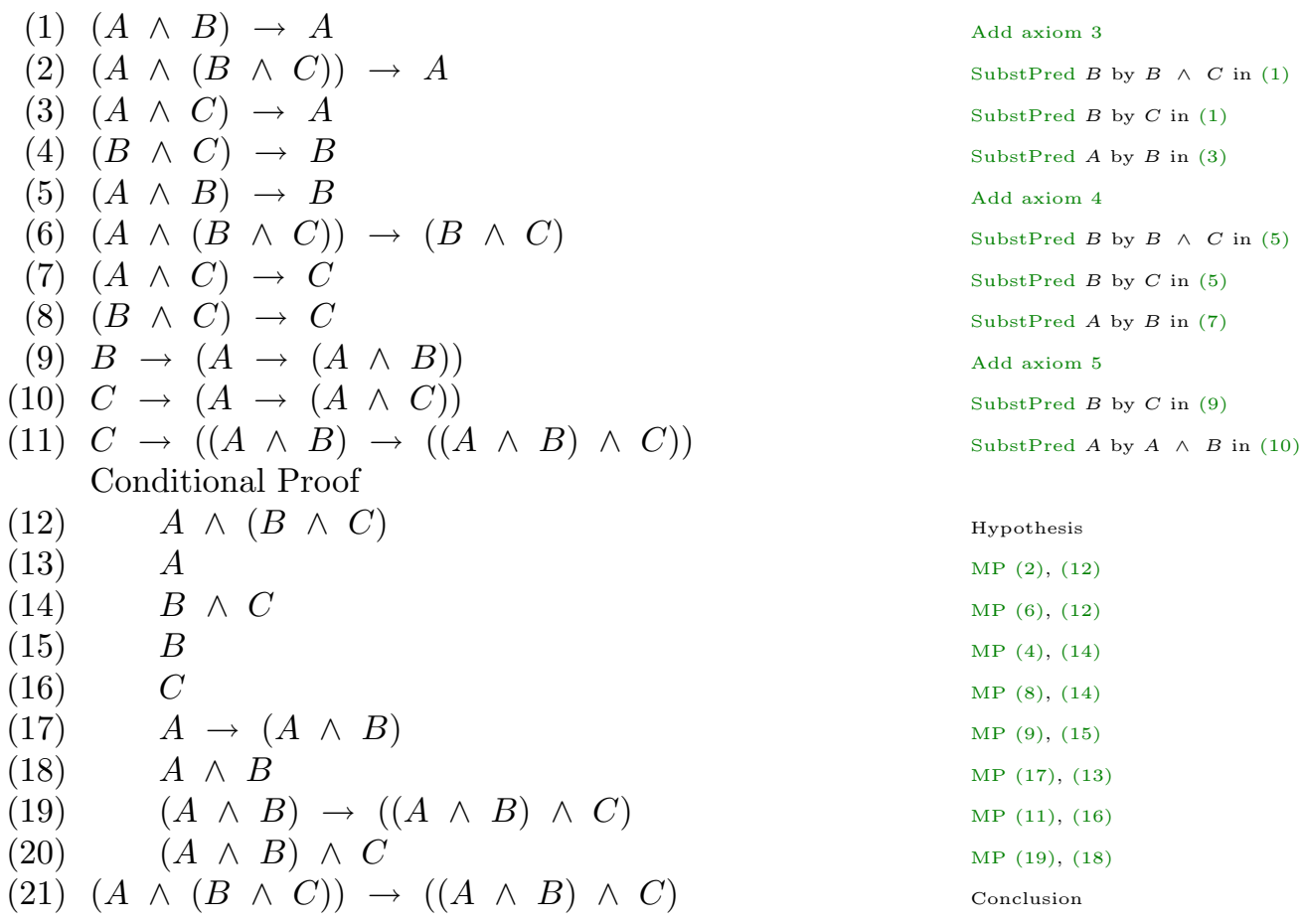

\subsection{Propositions about disjunction}

The disjunction is our theme here.

Proposition 21.

$$
(A \vee(B \vee C)) \rightarrow((A \vee B) \vee C)
$$

Proof.
(1) $A \rightarrow(A \vee B)$
(2) $A \rightarrow(A \vee C)$
(3) $(A \vee B) \rightarrow((A \vee B) \vee C)$ Conditional Proof
(4) $A$
(5) $\quad A \vee B$
(6) $\quad(A \vee B) \vee C$
(7) $A \rightarrow((A \vee B) \vee C)$
(8) $A \rightarrow(B \vee A)$
(9) $C \rightarrow(B \vee C)$
(10) $C \rightarrow(A \vee C)$
(11) $B \rightarrow(A \vee B)$
Conditional Proof

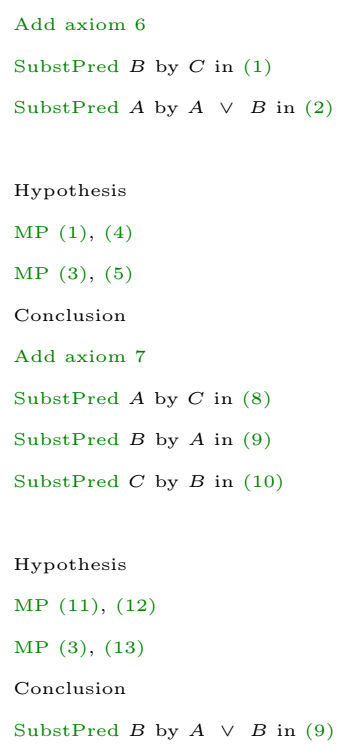
(12) $B$
(13) $A \vee B$
(14) $(A \vee B) \vee C$
(15) $B \rightarrow((A \vee B) \vee C)$
(16) $C \rightarrow((A \vee B) \vee C)$
SubstPred $B$ by $A \vee B$ in (9) 


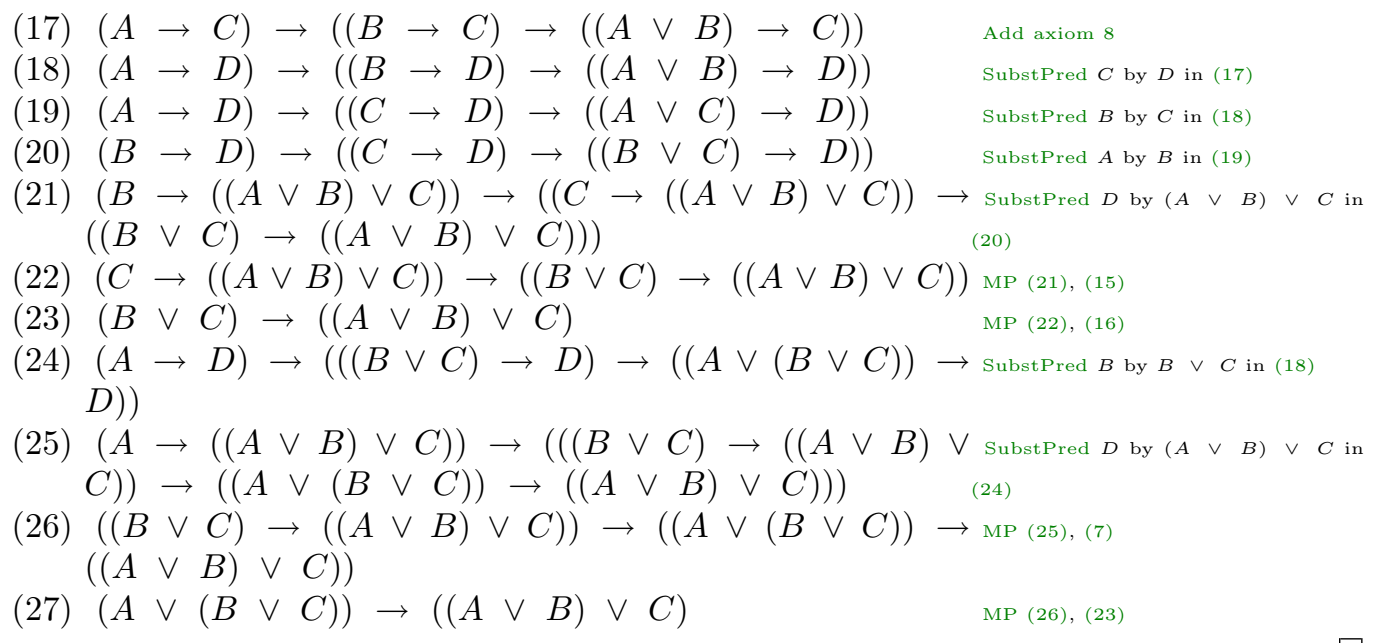

Proposition 22. [proposition: implication41]

$$
((A \vee B) \vee C) \rightarrow(A \vee(B \vee C))
$$

Proof.

(1) $A \rightarrow(A \vee B)$

(2) $A \rightarrow(A \vee(B \vee C))$

(3) $A \rightarrow(A \vee C)$

(4) $B \rightarrow(B \vee C)$

(5) $A \rightarrow(B \vee A)$

(6) $A \rightarrow(D \vee A)$

(7) $(B \vee C) \rightarrow(D \vee(B \vee C))$

(8) $(B \vee C) \rightarrow(A \vee(B \vee C))$ Conditional Proof

(9) $B$

(10) $B \vee C$

(11) $\quad A \vee(B \vee C)$

(12) $B \rightarrow(A \vee(B \vee C))$

(13) $C \rightarrow(B \vee C)$

Conditional Proof

(14) $\quad C$

(15) $B \vee C$

(16) $\quad A \vee(B \vee C)$

(17) $C \rightarrow(A \vee(B \vee C))$

(18) $(A \rightarrow C) \rightarrow((B \rightarrow C) \rightarrow((A \vee B) \rightarrow C))$

(19) $(A \rightarrow(A \vee(B \vee C))) \rightarrow((B \rightarrow(A \vee(B \vee C))) \rightarrow$ SubstPred $C$ by $A \vee(B \vee C)$ in $((A \vee B) \rightarrow(A \vee(B \vee C))))$

SubstPred $B$ by $B \vee C$ in (1)

SubstPred $B$ by $C$ in (1)

SubstPred $A$ by $B$ in (3)

Add axiom 7

SubstPred $B$ by $D$ in (5)

SubstPred $A$ by $B \vee C$ in (6)

SubstPred $D$ by $A$ in (7)

Hypothesis

MP (4), (9)

MP (8), (10)

Conclusion

SubstPred $A$ by $C$ in (5)

Hypothesis

MP (13), (14)

MP (8), (15)

Conclusion (18)

$(20)(B \rightarrow(A \vee(B \vee C))) \rightarrow((A \vee B) \rightarrow(A \vee(B \vee C)))$ мР (19), (2)

$(21)(A \vee B) \rightarrow(A \vee(B \vee C))$

$(22)(A \rightarrow D) \rightarrow((B \rightarrow D) \rightarrow((A \vee B) \rightarrow D)) \quad$ SubstPred $C$ by $D$ in (18)

(23) $(A \rightarrow D) \rightarrow((C \rightarrow D) \rightarrow((A \vee C) \rightarrow D))$

SubstPred $B$ by $C$ in (22)

$(24)((A \vee B) \rightarrow D) \rightarrow((C \rightarrow D) \rightarrow(((A \vee B) \vee C) \rightarrow$ SubstPred $A$ by $A \vee B$ in (23) $D))$

$(25) \quad((A \vee B) \rightarrow(A \vee(B \vee C))) \rightarrow((C \rightarrow(A \vee(B \vee$ SubstPred $D$ by $A \vee(B \vee C)$ in $C))) \rightarrow(((A \vee B) \vee C) \rightarrow(A \vee(B \vee C))))$

(24)

(26) $(C \rightarrow(A \vee(B \vee C))) \rightarrow(((A \vee B) \vee C) \rightarrow$ MP (25), (21) $(A \vee(B \vee C)))$

(27) $((A \vee B) \vee C) \rightarrow(A \vee(B \vee C))$

MP (26), (17) 
Proposition 23.

$$
(A \rightarrow B) \rightarrow((A \vee C) \rightarrow(B \vee C))
$$

Proof.

(1) $(A \rightarrow C) \rightarrow((B \rightarrow C) \rightarrow((A \vee B) \rightarrow C)) \quad$ Add axiom 8

(2) $(A \rightarrow D) \rightarrow((B \rightarrow D) \rightarrow((A \vee B) \rightarrow D)) \quad$ SubstPred $C$ by $D$ in (1)

(3) $(A \rightarrow D) \rightarrow((C \rightarrow D) \rightarrow((A \vee C) \rightarrow D)) \quad$ SubstPred $B$ by $C$ in (2)

(4) $(A \rightarrow(B \vee C)) \rightarrow((C \rightarrow(B \vee C)) \rightarrow((A \vee C) \rightarrow$ SubstPred $D$ by $B \vee C$ in (3) $(B \vee C)))$

(5) $A \rightarrow(A \vee B)$

(6) $A \rightarrow(A \vee C)$

Add axiom 6

(7) $B \rightarrow(B \vee C)$

SubstPred $B$ by $C$ in (5)

SubstPred $A$ by $B$ in (6)

(8) $A \rightarrow(B \vee A)$

Add axiom 7

(9) $C \rightarrow(B \vee C)$

SubstPred $A$ by $C$ in (8)

Conditional Proof

(10) $\quad A \rightarrow B$

Hypothesis

Conditional Proof

(13) $B \vee C$

(14) $\quad A \rightarrow(B \vee C)$

$(15) \quad(C \rightarrow(B \vee C)) \rightarrow((A \vee C) \rightarrow(B \vee C))$

(16) $\quad(A \vee C) \rightarrow(B \vee C)$

(17) $(A \rightarrow B) \rightarrow((A \vee C) \rightarrow(B \vee C))$

Hypothesis

MP (10), (11)

MP (7), (12)

Conclusion

MP (4), (14)

MP (15), (9)

Conclusion

\subsection{Propositions about negation}

Now we look at negation. Here we must use the principle of the excluded middle for the first time.

Proposition 24. [proposition:implication50]

$$
A \rightarrow \neg \neg A
$$

Proof.

(1) $A \rightarrow(B \rightarrow A)$

(2) $A \rightarrow(\neg A \rightarrow A)$

(3) $(A \rightarrow B) \rightarrow((A \rightarrow \neg B) \rightarrow \neg A)$

(4) $(\neg A \rightarrow B) \rightarrow((\neg A \rightarrow \neg B) \rightarrow \neg \neg A)$

(5) $(\neg A \rightarrow A) \rightarrow((\neg A \rightarrow \neg A) \rightarrow \neg \neg A)$

(6) $A \rightarrow A$

(7) $\neg A \rightarrow \neg A$

Conditional Proof

(8) $A$

(9) $\quad \neg A \rightarrow A$

(10) $\quad(\neg A \rightarrow \neg A) \rightarrow \neg \neg A$

(11) $\quad \neg \neg A$

(12) $A \rightarrow \neg \neg A$

Add axiom 1

SubstPred $B$ by $\neg A$ in (1)

Add axiom 9

SubstPred $A$ by $\neg A$ in (3)

SubstPred $B$ by $A$ in (4)

Add proposition 1

SubstPred $A$ by $\neg A$ in (6)

Hypothesis

MP (2), (8)

MP (5), (9)

MP (10), (7)

Conclusion 
Proposition 25. [proposition: implication51]

$$
(A \rightarrow \neg B) \rightarrow(B \rightarrow \neg A)
$$

Proof.
(1) $A \rightarrow(B \rightarrow A)$
(2) $C \rightarrow(B \rightarrow C)$
Add axiom
(3) $C \rightarrow(A \rightarrow C)$
(4) $B \rightarrow(A \rightarrow B)$
(5) $(A \rightarrow B) \rightarrow((A \rightarrow \neg B) \rightarrow \neg A)$
Conditional Proof
(6) $\quad A \rightarrow \neg B$
Conditional Proof

$$
B
$$$$
A \rightarrow B
$$$$
(A \rightarrow \neg B) \rightarrow \neg A
$$$$
\neg A
$$
(11) $\quad B \rightarrow \neg A$
(12) $(A \rightarrow \neg B) \rightarrow(B \rightarrow \neg A)$
SubstPred $A$ by $C$ in (1)
SubstPred $B$ by $A$ in (2)
SubstPred $C$ by $B$ in (3)
Add axiom 9
Hypothesis
Hypothesis
MP (4), (7)
MP (5), (8)
MP (9), (6)
Conclusion
Conclusion

Proposition 26. [proposition: implication52]

$$
(A \rightarrow B) \rightarrow(\neg B \rightarrow \neg A)
$$

Proof.
(1) $A \rightarrow(B \rightarrow A)$
(2) $C \rightarrow(B \rightarrow C)$
Add axiom
(3) $C \rightarrow(A \rightarrow C)$
(4) $\neg B \rightarrow(A \rightarrow \neg B)$
(5) $(A \rightarrow B) \rightarrow((A \rightarrow \neg B) \rightarrow \neg A)$
Conditional Proof
(6) $\quad A \rightarrow B$
(7) $\quad(A \rightarrow \neg B) \rightarrow \neg A$
Conditional Proof
$\begin{array}{ll}(8) & \neg B \\ \text { (9) } & A \rightarrow \neg B\end{array}$

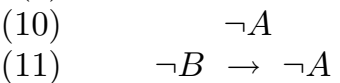
$\neg B \rightarrow \neg A$
$(12)(A \rightarrow B) \rightarrow(\neg B \rightarrow \neg A)$
SubstPred $A$ by $C$ in (1)
SubstPred $B$ by $A$ in (2)
SubstPred $C$ by $\neg B$ in (3)
Add axiom 9
Hypothesis
MP (5), (6)
Hypothesis
MP (4), (8)
MP (7), (9)
Conclusion
Conclusion

Proposition 27. [proposition: implication54]

$$
\neg \neg \neg A \rightarrow \neg A
$$

Proof.
(1) $A \rightarrow \neg \neg A$
(2) $(A \rightarrow B) \rightarrow(\neg B \rightarrow \neg A)$
(3) $(A \rightarrow \neg \neg A) \rightarrow(\neg \neg \neg A \rightarrow \neg A)$
(4) $\neg \neg \neg A \rightarrow \neg A$

Add proposition 24

Add proposition 26

SubstPred $B$ by $\neg \neg A$ in (2)

MP (3), (1) 
Proposition 28. [proposition:implication55]

$$
(\neg A \rightarrow A) \rightarrow \neg \neg A
$$

Proof.

(1) $A \rightarrow A$

(2) $\neg A \rightarrow \neg A$

(3) $(A \rightarrow B) \rightarrow((A \rightarrow \neg B) \rightarrow \neg A)$

(4) $(\neg A \rightarrow B) \rightarrow((\neg A \rightarrow \neg B) \rightarrow \neg \neg A)$

(5) $(\neg A \rightarrow A) \rightarrow((\neg A \rightarrow \neg A) \rightarrow \neg \neg A)$ Conditional Proof

(6) $\quad \neg A \rightarrow A$

(7) $\quad(\neg A \rightarrow \neg A) \rightarrow \neg \neg A$

(8) $\neg \neg A$

(9) $(\neg A \rightarrow A) \rightarrow \neg \neg A$

Add proposition 1

SubstPred $A$ by $\neg A$ in (1)

Add axiom 9

SubstPred $A$ by $\neg A$ in (3) SubstPred $B$ by $A$ in (4)

Hypothesis

MP (5), (6)

MP (7), (2)

Conclusion

Proposition 29.

$$
\neg \neg A \rightarrow A
$$

Proof.

(1) $A \vee \neg A$

(2) $(A \rightarrow C) \rightarrow((B \rightarrow C) \rightarrow((A \vee B) \rightarrow C))$

Add axiom 11

$(3)(A \rightarrow A) \rightarrow((B \rightarrow A) \rightarrow((A \vee B) \rightarrow A))$

Add axiom 8

(4) $A \rightarrow A$

(5) $(B \rightarrow A) \rightarrow((A \vee B) \rightarrow A)$

(6) $(\neg A \rightarrow A) \rightarrow((A \vee \neg A) \rightarrow A)$

(7) $\neg A \rightarrow(A \rightarrow B)$

(8) $\neg \neg A \rightarrow(\neg A \rightarrow B)$

(9) $\neg \neg A \rightarrow(\neg A \rightarrow A)$

Conditional Proof

(10) $\neg \neg A$

(11) $\neg A \rightarrow A$

(12) $\quad(A \vee \neg A) \rightarrow A$

(13) $A$

(14) $\neg \neg A \rightarrow A$

SubstPred $C$ by $A$ in (2) Add proposition 1

MP (3), (4)

SubstPred $B$ by $\neg A$ in (5)

Add axiom 10

SubstPred $A$ by $\neg A$ in (7) SubstPred $B$ by $A$ in (8)

Proposition 30.

$$
(\neg B \rightarrow \neg A) \rightarrow(A \rightarrow B)
$$

Proof.

(1) $\neg \neg A \rightarrow A$

(2) $\neg \neg B \rightarrow B$

(3) $(A \rightarrow \neg B) \rightarrow(B \rightarrow \neg A)$

(4) $(C \rightarrow \neg B) \rightarrow(B \rightarrow \neg C)$

(5) $(C \rightarrow \neg A) \rightarrow(A \rightarrow \neg C)$

(6) $(\neg B \rightarrow \neg A) \rightarrow(A \rightarrow \neg \neg B)$

Conditional Proof

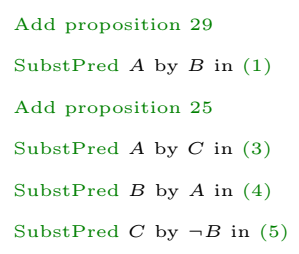



(7) $\neg B \rightarrow \neg A$
Hypothesis
(8) $\quad A \rightarrow \neg \neg B$
MP (6), (7)
(9) $A$
(10) $\quad \neg \neg B$
(11) $B$
$\rightarrow B$
Hypothesis
MP (8), (9)
(12) $\quad A \rightarrow B$
MP (2), (10)
Conclusion
(13) $(\neg B \rightarrow \neg A) \rightarrow(A \rightarrow B)$
Conclusion

\subsection{Mixing conjunction and disjunction.}

Now we show how disjunction and conjunction are connected.

Proposition 31. [proposition: implication70]

$$
((A \rightarrow C) \wedge(B \rightarrow C)) \rightarrow((A \vee B) \rightarrow C)
$$

Proof.

(1) $(A \rightarrow(B \rightarrow C)) \rightarrow((A \wedge B) \rightarrow C) \quad$ Add proposition 15

$(2)(A \rightarrow(B \rightarrow D)) \rightarrow((A \wedge B) \rightarrow D) \quad$ SubstPred $C$ by $D$ in (1)

(3) $((A \rightarrow C) \rightarrow(B \rightarrow D)) \rightarrow(((A \rightarrow C) \wedge B) \rightarrow D)$ SubstPred $A$ by $A \rightarrow C$ in (2)

(4) $((A \rightarrow C) \rightarrow((B \rightarrow C) \rightarrow D)) \rightarrow(((A \rightarrow$ SubstPred $B$ by $B \rightarrow C$ in (3) $C) \wedge(B \rightarrow C)) \rightarrow D)$

$(5)((A \rightarrow C) \rightarrow((B \rightarrow C) \rightarrow((A \vee B) \rightarrow C))) \rightarrow$ SubstPred $D$ by $(A \vee B) \rightarrow C$ in $(((A \rightarrow C) \wedge(B \rightarrow C)) \rightarrow((A \vee B) \rightarrow C))$

(6) $(A \rightarrow C) \rightarrow((B \rightarrow C) \rightarrow((A \vee B) \rightarrow C)) \quad$ Add axiom 8

(7) $((A \rightarrow C) \wedge(B \rightarrow C)) \rightarrow((A \vee B) \rightarrow C) \quad$ MP (5), (6)

Proposition 32. [proposition: imp1ication71]

$$
((A \wedge B) \vee C) \rightarrow((A \vee C) \wedge(B \vee C))
$$

Proof.

(1) $B \rightarrow(A \rightarrow(A \wedge B))$

(2) $(B \vee C) \rightarrow(A \rightarrow(A \wedge(B \vee C)))$

(3) $(B \vee C) \rightarrow((A \vee C) \rightarrow((A \vee C) \wedge(B \vee C)))$

(4) $(A \wedge B) \rightarrow A$

$(5)(A \rightarrow B) \rightarrow((A \vee C) \rightarrow(B \vee C))$

(6) $(A \rightarrow D) \rightarrow((A \vee C) \rightarrow(D \vee C))$

(7) $((A \wedge B) \rightarrow D) \rightarrow(((A \wedge B) \vee C) \rightarrow(D \vee C))$

Add axiom 5

(8) $((A \wedge B) \rightarrow A) \rightarrow(((A \wedge B) \vee C) \rightarrow(A \vee C))$

(9) $((A \wedge B) \vee C) \rightarrow(A \vee C)$

SubstPred $B$ by $B \vee C$ in (1)

(10) $(A \wedge B) \rightarrow B$

(11) $((A \wedge B) \rightarrow B) \rightarrow(((A \wedge B) \vee C) \rightarrow(B \vee C))$

(12) $((A \wedge B) \vee C) \rightarrow(B \vee C)$

Conditional Proof

(13) $\quad(A \wedge B) \vee C$

(14) $A \vee C$

(15) $B \vee C$

SubstPred $A$ by $A \vee C$ in (2)

Add axiom 3

Add proposition 23

SubstPred $B$ by $D$ in (5)

(16)

$$
(A \vee C) \rightarrow((A \vee C) \wedge(B \vee C))
$$

Hypothesis

MP (9), (13)

MP (12), (13)

MP (3), (15) 
(17) $\quad(A \vee C) \wedge(B \vee C)$

(18) $((A \wedge B) \vee C) \rightarrow((A \vee C) \wedge(B \vee C))$

MP (16), (14)

Conclusion

Proposition 33.

$$
((A \vee C) \wedge(B \vee C)) \rightarrow((A \wedge B) \vee C)
$$

Proof.
(1) $A \rightarrow(B \vee A)$
(2) $C \rightarrow(B \vee C)$
(3) $C \rightarrow((A \wedge B) \vee C)$
(4) $(A \rightarrow C) \rightarrow((B \rightarrow C) \rightarrow((A \vee B) \rightarrow C))$
$(5)(A \rightarrow D) \rightarrow((B \rightarrow D) \rightarrow((A \vee B) \rightarrow D))$
$(6)(A \rightarrow D) \rightarrow((C \rightarrow D) \rightarrow((A \vee C) \rightarrow D))$
Add axiom 7
SubstPred $A$ by $C$ in (1)
SubstPred $B$ by $A \wedge B$ in (2)
Add axiom 8
$(7)(B \rightarrow D) \rightarrow((C \rightarrow D) \rightarrow((B \vee C) \rightarrow D))$
SubstPred $C$ by $D$ in (4)
SubstPred $B$ by $C$ in (5)
(8) $(B \rightarrow((A \wedge B) \vee C)) \rightarrow((C \rightarrow((A \wedge B) \vee C)) \rightarrow$ $((B \vee C) \rightarrow((A \wedge B) \vee C)))$
(9) $\quad$ C

$$
\begin{aligned}
& C \\
& \text { Conditional Proof }
\end{aligned}
$$
SubstPred $A$ by $B$ in (6)
(10)$$
B
$$$$
(A \wedge B) \vee C
$$
(12) $B \rightarrow((A \wedge B) \vee C)$
$(13) \quad(C \rightarrow((A \wedge B) \vee C)) \rightarrow((B \vee C) \rightarrow$ MP (8), (12) $((A \wedge B) \vee C))$
(14) $\quad(B \vee C) \rightarrow((A \wedge B) \vee C)$
(15) $C \rightarrow((B \vee C) \rightarrow((A \wedge B) \vee C))$
(16) $(A \rightarrow B) \rightarrow((A \vee C) \rightarrow(B \vee C))$
$(17)(D \rightarrow B) \rightarrow((D \vee C) \rightarrow(B \vee C))$
$(18)(D \rightarrow(A \wedge B)) \rightarrow((D \vee C) \rightarrow((A \wedge B) \vee C))$ SubstPred $B$ by $A \wedge B$ in (17)
$(19)(B \rightarrow(A \wedge B)) \rightarrow((B \vee C) \rightarrow((A \wedge B) \vee C))$ SubstPred $D$ by $B$ in (18)
(20) $A \rightarrow(B \rightarrow(A \wedge B))$
Add proposition 10 Conditional Proof
$\begin{array}{ll}(21) & A \\ (22) & B \rightarrow(A \wedge B)\end{array}$
$\begin{array}{ll}(22) & B \rightarrow(A \wedge B) \\ (23) & (B \vee C) \rightarrow((A \wedge B) \vee C)\end{array}$
(24) $A \rightarrow((B \vee C) \rightarrow((A \wedge B) \vee C))$
$(25)(A \rightarrow C) \rightarrow((B \rightarrow C) \rightarrow((A \vee B) \rightarrow C))$
$(26)(A \rightarrow D) \rightarrow((B \rightarrow D) \rightarrow((A \vee B) \rightarrow D))$
MP (13), (3)
Conclusion
Add proposition 23
$(27)(A \rightarrow D) \rightarrow((C \rightarrow D) \rightarrow((A \vee C) \rightarrow D))$
Hypothesis
MP (20), (21)
MP (19), (22)
Conclusion
Add axiom 8
$(28)(A \rightarrow((B \vee C) \rightarrow((A \wedge B) \vee C))) \rightarrow((C \rightarrow$ SubstPred $D$ by $(B \vee C) \rightarrow((A \wedge$ $((B \vee C) \rightarrow((A \wedge B) \vee C))) \rightarrow((A \vee C) \rightarrow B) \vee C)$ in (27) $((B \vee C) \rightarrow((A \wedge B) \vee C))))$
(29) $(C \rightarrow((B \vee C) \rightarrow((A \wedge B) \vee C))) \rightarrow\left((A \vee C) \rightarrow \mathrm{MP}_{(28),(24)}\right.$ $((B \vee C) \rightarrow((A \wedge B) \vee C)))$
$(30)(A \vee C) \rightarrow((B \vee C) \rightarrow((A \wedge B) \vee C)) \quad$ MP (29), (15)
$(31)(A \rightarrow(B \rightarrow C)) \rightarrow((A \wedge B) \rightarrow C) \quad$ Add proposition 15
$(32)(A \rightarrow(B \rightarrow D)) \rightarrow((A \wedge B) \rightarrow D) \quad$ SubstPred $C$ by $D$ in (31)
$(33)((A \vee C) \rightarrow(B \rightarrow D)) \rightarrow(((A \vee C) \wedge B) \rightarrow D)$ SubstPred $A$ by $A \vee C$ in (32)
$(34)((A \vee C) \rightarrow((B \vee C) \rightarrow D)) \rightarrow(((A \vee C) \wedge(B \vee$ SubstPred $B$ by $B \vee C$ in (33) $C)) \rightarrow D)$
(35) $((A \vee C) \rightarrow((B \vee C) \rightarrow((A \wedge B) \vee C))) \rightarrow \operatorname{SubstPred} D$ by $(A \wedge B) \vee C$ in $(((A \vee C) \wedge(B \vee C)) \rightarrow((A \wedge B) \vee C))$
(36) $((A \vee C) \wedge(B \vee C)) \rightarrow((A \wedge B) \vee C) \quad$ мP (35), (30) 


\section{Bibliography}

[1] P.S. Novikov, Elements of Mathematical Logic, Edinburgh: Oliver and Boyd, 1964. 5

[2] V. Detlovs, K. Podnieks, Introduction to Mathematical Logic, https:// dspace.lu.lv/dspace/handle/7/1308. 5

[3] D. Hilbert, W. Ackermann, Grundzüge der theoretischen Logik, 2nd ed., Berlin: Springer, 1938. English version: Principles of Mathematical Logic, Chelsea, New York 1950, ed. by R. E. Luce. See also http://www.math. uwaterloo.ca/ snburris/htdocs/scav/hilbert/hilbert.html 5, 13

[4] E. Mendelson, Introduction to Mathematical Logic, 3rd. ed., Belmont, CA: Wadsworth, 1987.

[5] qedeq_logic_v1 http://www.qedeq.org/0_04_07/doc/math/qedeq_ logic_v1.xml 


\section{Index}

arity, 7

axiom

of existential generalization, 12 of universal instantiation, 12 axioms, 11

bound subject variable, 8

calculus

propositional, 9

conjunction

elimination, 11

introduction, 11

constant

function, 7

individual, 8

predicate, 7

deduction theorem, 16

disjunction

elimination, 11

introduction, 11

equivalence

elimination, 12

introduction, 12

existential quantifier, 8

formula, 7,8

part, 9

function constant, 7

function variable, 7

hypothesis distribution, 11

implication introduction, 11

individual constant, 8

Modus Ponens, 12

negation

elimination, 12

excluded middle, 12

introduction, 12

part formula, 9

predicate constant, 7

predicate variable, 7

proposition variable, 7

propositional calculus, 9 quantifier

existential, 8

scope, 9

universal, 8

rank, 7

rules

of inference, 12

of predicate calculus, 12

scope, 9

sentence letters, 7

subject variable, 7

bound, 8

free, 8

summary, 5

term, 7,8

universal quantifier, 8

variable

function, 7

predicate, 7

proposition, 7

subject, 7 